\title{
Advances in the management of male infertility
}

\section{Gerhard Haidl}

Address: Department of Dermatology/Andrology Unit, University of Bonn, Sigmund-Freud-Str 25, 53105 Bonn, Germany

Email: gerhard.haidl@ukb.uni-bonn.de

Fl000 Medicine Reports 2009, I:92 (doi:10.3410/MI-92)

The electronic version of this article is the complete one and can be found at: http://FI000.com/Reports/Medicine/content/I/92

\begin{abstract}
Male infertility can be treated by surgical procedures (e.g., varicocelectomy) or by administration of drugs if causal factors (e.g., seminal tract infections) are detected. In more severe cases, methods of assisted fertilization often have to be applied, but even these have only a limited success rate. Recent studies have demonstrated that disturbances of sperm DNA integrity (determined by the acridine orange test) can explain certain cases of fertilization failure and failure to achieve pregnancy following in vitro fertilisation with intracytoplasmic sperm injection. The evaluation of DNA integrity should be considered when diagnosing male infertility as it has been shown to be an independent factor and can be used as a supplement to standard semen analysis. Analysis of DNA integrity may, therefore, provide further information about altered male fertility and lead to administration of more appropriate therapy.
\end{abstract}

\section{Introduction and context}

DNA integrity is a prerequisite, not only for the fertilization of an oocyte, but also for oocyte activation and achievement of an ongoing pregnancy [1]. It has been documented that there is a negative correlation between defective sperm chromatin structure (DNA breaks) and fertility, in vivo and in vitro [2]. Several studies have demonstrated that disturbed DNA integrity reduces fertilization and pregnancy rate during in vitro fertilisation (IVF) and intracytoplasmic sperm injection (ICSI) treatment, and also increases the risk of early miscarriage $[3,4]$.

DNA integrity can be assessed by the sperm chromatin structure assay (SCSA), by terminal deoxynucleotidyl transferase-mediated dUTP nick end labelling (TUNEL), and more recently, the sperm chromatin dispersion assay. Special consideration should be made when comparing these methods, however, because they are each measuring different parameters. The SCSA is considered an indirect indicator of DNA damage. It is based on exposing sperm nuclei to acid then staining them with acridine orange (the acid exposure causes denaturation of double-stranded DNA in sperm with impaired chromatin structure). The stain will attach to the ends of the broken DNA only, enabling evaluation of the proportion of single- and double-stranded DNA in the sample. The sperm chromatin dispersion assay also measures the susceptibility of sperm DNA to acid denaturation, but in a different way. The SCSA is based on the metachromatic property of the stain acridine orange, where intact DNA are characterized by a green fluorescence and disturbed DNA by orange fluorescence. The evaluation is carried out by flow cytometry; therefore a high number of cells (about 10,000) can be studied. The sperm chromatin dispersion test is performed using conventional bright field microscopy and analyses dispersed DNA loop halos. Between 300 and 500 spermatozoa can be evaluated during this test. The TUNEL assay uses fluorescein-dUTP to label single- and double-stranded DNA breaks.[5-7].

\section{Recent advances}

In most recent studies, no correlation between DNA integrity and conventional semen parameters has been found; therefore, DNA integrity can be considered as a sperm function that is independent from sperm count, motility, and morphology $[7,8]$. 
Disturbances in sperm DNA integrity have been described in patients with varicoceles, testicular tumours, genital tract infections/inflammations, and patients with 'idiopathic' infertility $[1,9,10]$. Disturbances of DNA integrity occur predominatly at the post-testicular level, which is why a recent study suggested testicular sperm extraction with subsequent ICSI as a possible treatment [11].

Research by different groups suggests that extrinsic oxidative stress, including environmental or industrial toxins or smoking, seems to be the major cause of sperm DNA damage rather than apoptosis [5], and a recent study showed that treatment with antioxidants has positive effects on implantation and pregnancy rates after ICSI [12].

Regarding further treatment options for disturbed DNA integrity, it is fortunate that a considerable number of recent studies have focused on the correlation between disturbed DNA integrity and genital tract inflammations and infections, respectively. It was shown that DNA integrity was impaired in leukocytospermic semen samples $[13,14]$, and in patients with less than 1 million leukocytes per $\mathrm{ml}$ and high levels of reactive oxygen species [5]. In the last scenario, disturbed DNA integrity may still allow oocyte fertilization and formation of the 2-pronuclei stage, but when the paternal genes are 'switched on' at the 4-cell stage, further embryonic development stops because of oxidation and fragmentation of the paternal DNA, even after blastocyst formation [5].

Another recent advance is the demonstration that DNA integrity is improved after antibiotic treatment in patients infected with Chlamydia trachomatis [15]. Another recent study demonstrated that, compared with men without varicocele, men with varicocele have a higher percentage of sperm cells with DNA fragmentation. Thus, evaluation of sperm DNA fragmentation could be an important factor in deciding treatment options for men with varicocele [9]. This is supported by further studies, including a small one in which 10 of 11 patients with varicocele showed a significant decrease in sperm DNA fragmentation after varicocele repair, and an earlier, larger study of 37 patients $[16,17]$.

\section{Implications for the clinical practice}

The evaluation of DNA integrity should be considered when diagnosing male infertility. However, the different DNA integrity tests can often draw different conclusions and sometimes even the same test can yield different results (most probably due to low study populations), therefore, interpretations of test results in clinical practice have to be made with some caution. A greater quantity of larger studies is needed for validation of such tests before they are ready to be used in general clinical practice. Despite these limitations, this is an interesting and promising field that may offer new tools for andrological diagnostic work-up and may finally lead to more diagnosis-related instead of symptoms-related treatment of male infertility.

\section{Abbreviations}

ICSI, intracytoplasmic sperm injection; IVF, in vitro fertilisation; SCSA, sperm chromatin structure assay; TUNEL, terminal deoxynucleotidyl transferase-mediated dUTP nick end labelling.

\section{Competing interests}

The author declares that he has no competing interests.

\section{References}

I. Evenson D, Wixon R: Meta-analysis of sperm DNA fragmentation using the sperm chromatin structure assay. Reprod Biomed Online 2006, I 2:466-72.

2. Virro MR, Larson-Cook KL, Evenson DP: Sperm chromatin structure assay (SCSA) parameters are related to fertilization, blastocyst development, and ongoing pregnancy in in vitro fertilization and intracytoplasmic sperm injection cycles. Fertil Steril 2004, 8 I: I 289-95.

3. Lin MH, Kuo-Kuang Lee R, Li SH, Lu CH, Sun FJ, Hwu YM: Sperm chromatin structure assay parameters are not related to fertilization rates, embryo quality, and pregnancy rates in in vitro fertilization and intracytoplasmic sperm injection, but might be related to spontaneous abortion rates. Fertil Steril 2008, 90:352-9.

4. Carrell DT, Liu L, Peterson CM, Jones KP, Hatasaka HH, Erickson L, Campbell B: Sperm DNA fragmentation is increased in couples with unexplained recurrent pregnancy loss. Arch Androl 2003, 49:49-55.

5. Henkel R, Hajimohammad M, Stalf T, Hoogendijk C, Mehnert C, Menkveld R, Gips H, Schill WB, Kruger TF: Influence of deoxyribonucleic acid damage on fertilization and pregnancy. Fertil Steril 2004, 81:965-72.

6. Muriel L, Garrido N, Fernández JL, Remohí J, Pellicer A, de los Santos M], Meseguer M: Value of the sperm deoxyribonucleic acid fragmentation level, as measured by the sperm chromatin dispersion test, in the outcome of in vitro fertilization and intracytoplasmic sperm injection. Fertil Steril 2006, 85:37I-83.

7. Bungum M, Humaidan P, Axmon A, Spano M, Bungum L, Erenpreiss J, Giwercman A: Sperm DNA integrity assessment in prediction of assisted reproduction technology outcome. Hum Reprod 2007, 22:174-9.

FI000 Factor 3.0 Recommended Evaluated by Jay Sandlow 18 Apr 2007

8. Agarwal A, Allamaneni SS: Sperm DNA damage assessment: a test whose time has come. Fertil Steril 2005, 84:850-3.

9. Bertolla RP, Cedenho AP, Filho PAH, Lima SB, Ortiz V, Srougi M: Sperm nuclear DNA fragmentation in adolescents with varicocele. Fertil Steril 2006, 85:625-8.

10. Meseguer M, Santiso R, Garrido N, Fernandez JL: The effect of cancer on sperm DNA fragmentation as measured by the sperm chromatin dispersion test. Fertil Steril 2008, 90:225-7.

II. Greco E, Scarselli F, lacobelli M, Rienzi L, Ubaldi F, Ferrero S, Franco G, Anniballo N, Mendoza C, Tesarik J: Efficient treatment of infertility due to sperm DNA damage by ICSI with testicular spermatozoa. Hum Reprod 2005, 20:226-30. 
12. Greco E, Romano S, lacobelli M, Ferrero S, Baroni E, Minasi MG, Ubaldi F, Rienzi L, Tesarik J: ICSI in cases of sperm DNA damage: beneficial effect of antioxidant treatment. Hum Reprod 2005, 20:2590-4.

13. Erenpreiss J, Hlevicka S, Zalkalns J, Erenpreisa J: Effect of leukocytospermia on sperm DNA integrity: a negative effect in abnormal semen samples. J Androl 2002, 23:717-23.

14. Kopa Z, Wenzel J, Papp GK, Haidl G: Role of granulocyte elastase and interleukin- 6 in the diagnosis of male genital tract inflammation. Andrologia 2005, 37: I88-94.
15. Gallegos G, Ramos B, Santiso R, Goyanes V, Gosálvez J, Fernández JL: Sperm DNA fragmentation in infertile men with genitourinary infection by Chlamydia trachomatis and Mycoplasma. Fertil Steril 2008, 90:328-34.

16. Werthman P, Wixon R, Kasperson K, Evenson DP: Significant decrease in sperm deoxyribonucleic acid fragmentation after varicocelectomy. Fertil Steril 2008, 90:1800-4.

17. Zini A, Blumenfeld A, Libman J, Willis J: Beneficial effect of microsurgical varicocelectomy on human sperm DNA integrity. Hum Reprod 2005, 20:1018-21. 